\title{
Multi-period Optimal Energy Procurement and Demand Responses in Smart Grid with Uncertain Supply
}

\author{
Libin Jiang and Steven H. Low
}

\begin{abstract}
We propose a simple model that integrates multiperiod electricity markets, uncertainty in renewable generation, and real-time dynamic demand response. A load-serving entity decides its day-ahead procurement to optimize expected social welfare a day before energy delivery. At delivery time when renewable generation is realized, it sets prices to manage demand and purchase additional power on the real-time market, if necessary, to balance supply and demand. We derive the optimal dayahead decision, propose real-time demand response algorithm, and study the effect of volume and variability of renewable generation on these optimal decisions and on social welfare.
\end{abstract}

\section{MODEL}

Consider a set $N$ of households/users that are served by a single load-serving entity (LSE). The LSE participates in the wholesale electricity markets (day-ahead, real-time balancing, and ancillary services, etc) to purchase electricity from generators and then sell it to the users in the retail market. We aim to capture three major aspects of future smart grids: multitimescale energy procurement by the LSE, uncertain supply of renewable energy, and active demand management by the users. Our goal is to propose an approach to optimally integrate demand response and power procurement.

Consider a discrete-time model that models a day. Each day is divided into $T$ timeslots of equal duration, indexed by $t \in \mathscr{T}:=\{1,2, \cdots, T\}$. The duration of a timeslot can be 15 , 30 , or 60 mins, corresponding to the time resolution at which energy dispatch or demand response decisions are made.

The system operates as follows. By a certain time, say, noon of the day before delivery, the LSE decides the amount $P_{d}:=\left(P_{d}(t), t \in \mathscr{T}\right)$ of power to schedule on the day-ahead market for each period $t$ in the following day. This incurs a cost $c_{d}\left(P_{d}(t) ; t\right)$ for slot $t$. At this decision time, the information available to the LSE is the probability distribution of renewable production $P_{r}:=\left(P_{r}(t), t \in \mathscr{T}\right)$ in the next day; and the information available to user $i$ is his utility functions $u_{i}\left(x_{i}(t) ; t\right)$ where $x_{i}(t)$ is his consumption in slot $t$. By time $t^{-}$, we assume that the renewable-energy generation $P_{r}(t)$ have been realized. Then the users decide their actual demand $x_{i}(t) \in\left[\underline{x}_{i}(t), \bar{x}_{i}(t)\right]$ in slot $t$; and the LSE decides to use $P_{o}(t) \leq P_{d}(t)$ from the scheduled day-ahead power (which incurs an operation cost of $\left.c_{o}\left(P_{o}(t) ; t\right)\right)$, and purchases $P_{b}(t) \geq 0$ on the real-time market (with a cost $c_{b}\left(P_{b}(t) ; t\right)$ ), to satisfy the demand (i.e., such that $\left.P_{o}(t)+P_{b}(t)+P_{r}(t) \geq \sum_{i} x_{i}(t)\right)$. It is desirable to use as much

The authors are with the Division of Engineering \& Applied Science, Caltech. Emails: \{libinj, slow @ealtech.edu renewable power as possible; for simplicity, we assume $P_{r}(t)$ incurs no cost.

Assume that all cost functions are increasing and convex, and the utility functions are increasing and strictly concave. Our goal is to maximize the expected welfare, where the welfare is the total user utility minus the costs of supply.

\section{OPTIMAL ENERGY PROCUREMENT AND DEMAND RESPONSES}

In this extended abstract, we focus on the case without time correlation in demand. (The case with such correlation is discussed in [1].) In this case, maximizing the expected social welfare of the day reduces to independently maximizing the expected social welfare for each time slot. We consider slot $t$, and omit the notation $t$ for brevity. We derive the optimal solution using backwards induction.

At real time (i.e., at time $t^{-}$), $P_{d}$ for slot $t$ has been chosen, which incurs a cost of $c_{d}\left(P_{d}\right)$. Also, $P_{r}$ has been realized. So, the LSE cooperates with the users to solve the following problem

$$
\begin{aligned}
\tilde{W}\left(P_{d} ; P_{r}\right):= & \max _{x, P_{o}, P_{b}}\left\{\sum_{i} u_{i}\left(x_{i}\right)-c_{o}\left(P_{o}\right)-c_{b}\left(P_{b}\right)\right\} \\
\text { s.t. } \quad & x_{i} \leq x_{i} \leq \bar{x}_{i}, \forall i ; P_{o}, P_{b} \geq 0 \\
& P_{o} \leq P_{d} \\
& P_{r}+P_{o}+P_{b} \geq \sum_{i} x_{i} .
\end{aligned}
$$

Associate dual variables $\mu_{1}$ and $\mu_{2}$ with the constraint (1) and (2) respectively. The above problem can be solved in a distributed way by a primal-dual algorithm (Algorithm 2 in [1]). Let $\mu_{1}^{*}, \mu_{2}^{*}$ be the optimal dual variables (obtained after the algorithm converges), then $\mu_{2}^{*}$ is the real-time price the LSE imposes on the users.

So, if the LSE chooses $P_{d}$ as the day-ahead power, the expected welfare is $W\left(P_{d}\right):=E\left[\tilde{W}\left(P_{d} ; P_{r}\right)\right]-c_{d}\left(P_{d}\right)$. We show in [1] that $\tilde{W}\left(P_{d} ; P_{r}\right)$ is concave in $P_{d}$, and

$$
\partial \tilde{W}\left(P_{d} ; P_{r}\right) / \partial P_{d}=\mu_{1}^{*}
$$

if $\tilde{W}\left(P_{d} ; P_{r}\right)$ is differentiable at point $P_{d}$. Therefore, a stochastic subgradient algorithm the converges to the set of optimal $P_{d}$ is as follows. The algorithm is run one day in advance by simulating the real-time system.

Algorithm: Deciding the day-ahead power

1) Initially, let $P_{d}^{0}=0$.

2) In step $m+1=1,2, \ldots$, the LSE generates a sample of $P_{r}$ based on its distribution (denoted by $P_{r}^{m}$ ). Run Algorithm 
2 in [1] with the sample to find $\mu_{1}^{*}$, and denote it by $\mu_{1}^{* m}$. Then, compute

$$
P_{d}^{m+1}=\left\{P_{d}^{m}+\alpha^{m}\left[\mu_{1}^{* m}-c_{d}^{\prime}\left(P_{d}^{m}\right)\right]\right\}_{0}
$$

where $\alpha^{m}=1 /(m+1)$ is the step size.

\section{EFFECT OF RENEWABLE ENERGY ON SOCIAL WELFARE}

We investigate how the statistics of the renewable energy affects the maximal expected welfare $\max _{P_{d} \geq 0}\left\{W\left(P_{d}\right)\right\}$. Assume that the renewable energy is parametrized by $a, b>0$ as follows:

$$
P_{r}(a, b):=a \cdot \mu_{r}+b \cdot V_{r}
$$

where $\mu_{r}>0$ is a constant, and $V_{r}$ is a zero-mean random variable. So, $a$ and $b$ are indicators of the mean and variance of $P_{r}(a, b)$. Denote by $W^{*}\left(P_{r}(a, b)\right)$ the maximal expected welfare when the renewable energy is $P_{r}(a, b)$.

Proposition 1: (i) When $a$ is fixed, $W^{*}\left(P_{r}(a, b)\right)$ is nonincreasing with $b$; (ii) When $b$ is fixed, $W^{*}\left(P_{r}(a, b)\right)$ is nondecreasing with $a$; (iii) $W^{*}\left(P_{r}(s, s)\right)$ is non-decreasing with $s>0$, where $s$ is, for example, the size of the renewable energy farm.

\section{REFERENCES}

[1] L. Jiang and S.H. Low, "Multi-period Optimal Procurement and Demand Responses in the Presence of Uncertain Supply," Technical Report, Caltech, May 2011. Available: http://www.its.caltech.edu/ libinj/DR.pdf 